\title{
CONTRATO PSICOLÓGICO: FORMALIZAÇÃO DA LINGUAGEM
}

\author{
José Manuel Magno Lopes \\ Professor Adjunto Escola Superior de Tecnologia e Gestão - Politécnico de Leiria \\ magnolopes@gmail.com \\ Florencio Vicente Castro \\ Catedrático de Psicología \\ Facultad de Educación Universidad de Extremadura \\ https://doi.org/10.17060/ijodaep.2017.n1.v3.1004
}

Fecha de Recepción: 13 Enero 2017

Fecha de Admisión: 1 Abril 2017

\section{RESUMO}

Marco Conceptual: Um aspeto fundamental no estudo do contrato psicológico é proporcionar um amplo modelo de análise, pela compreensão das perceções, emoções e comportamentos dos indivíduos, bem como o momento e contextos em que aqueles ocorrem, com o propósito de intervir em relacionamentos que promovam um alto comprometimento entre as partes. Objetivos: A literatura corrente, de onde se destacam autores como Rousseau e Guest, apresenta alguns modelos do contrato psicológico aplicáveis em contextos específicos, assim como instrumentos metodológicos de acesso aos dados aplicáveis em contextos considerados para o efeito. Não descurando a conformidade de cada um importa desenvolver um modelo que registe, leia, interprete e devolva um sistema de informação que considere todas as formas de comunicação e não esteja dependente do tempo ou espaço para recolha de dados, ou seja, um sistema que registo qualquer forma de expressão do indivíduo a todo o tempo. Método: No âmbito de um Projeto que nos propomos desenvolver, este trabalho, em particular, é essencialmente de cariz qualitativo que estuda os requisitos da linguagem formal, com principal incidência de Chomsky, pelo desenvolvimento de uma gramática própria associada a um modelo de genérico de contrato psicológico pela sistematização da informação. Resultados: Tomamos como proposição que uma linguagem é composta por um conjunto de símbolos (léxico) que usa um conjunto de regras (sintaxe) para que seja percebida por uma determinado grupo. A análise e interpretação da semântica formal pode integrar um conjunto de regras de transformação que possam ser interpretados como regras válidas de inferência, um conjunto de axiomas ou a coexistência de ambos. Conclusão: Esta investigação procura enraizar um protocolo informal de comunicação através de uma estrutura formal de uma linguagem que interprete e dê significado semântico à simbologia da linguagem do contrato psicológico com o apoio de um sistema de informação. 


\section{CONTRATO PSICOLÓGICO: FORMALIZAÇÃO DA LINGUAGEM}

Palavras-chave: Contrato Psicológico, Perceção, Linguagens Formais.

\section{ABSTRACT}

Psychological Contract: Formalization of Language

Conceptual Framework: A fundamental aspect in the study of the psychological contract it to provide a broad model of analysis, by understanding the individual's perceptions, emotions and behaviour, as well as the moment and context in which they occur with the aim of intervening in relationships that promote a major commitment between the parties. Objectives: The current literature, in particular authors such as Rousseau and Guest, presents some models of psychological contract which are applicable in specific contexts as well as methodological instruments for the access to data which are applicable in contexts considered for this purpose. Without disregarding the conformity of each one, it is important to develop a model which registers, reads, interprets and provides an information system that considers all the means of communication and which is not dependent on time or space to collect data, i.e., a system that registers any of the individual's means of expression at all times. Method: Within a Project which we aim to carry out, this paper in particular is of an essentially qualitative nature and studies the requirements of formal language, with primary focus on Chomsky, for the development of a unique grammar which is associated to a generic model of psychological contract through the systematisation of information. Results: We based this study on the proposition that a language is composed of a set of symbols (lexis) which uses a set of rules (syntax) so that it can be understood by a specific group. The analysis and interpretation of formal semantics may include a set of transformation rules which can be interpreted as valid rules of inference, a set of axioms or the coexistence of both. Conclusion: This study aims to establish an informal communication protocol through the formal structure of a language which interprets and gives semantic meaning to the symbology of the language of the psychological contract with the aid of an information system.

Keywords: Psychological Contract, Perception, Formal Languages.

\section{MARCO CONCEPTUAL}

Os contratos psicológicos são enfatizados pela literatura como modelos mentais e flexíveis (Rousseau, 1995). As mensagens podem ter origem em diversas fontes e a sua interpretação depende, em bom rigor, de cada indivíduo, i.e., de esquemas mentais que podem condicionar a aceção do objeto, bem como a interpretação subjetiva que cada um faz acerca das obrigações que comete a si próprio e à outra parte no contrato psicológico - razão pela qual é conferido ao contrato psicológico uma natureza subjetiva, muito evidenciada por Rousseau (1989). Neste sentido, mesmo que o indivíduo acredite que a outra parte concorda com os termos do contrato, não é certo que esse entendimento tenha sido exatamente 0 mesmo (Côrtes \& Silva, 2006; Robinson \& Rousseau, 1994).

Como salientam Correia e Mainardes (2010), o contrato psicológico existe no "quadro interpretativo que cada indivíduo cria" (p.269), desenvolve e ajusta progressivamente na relação que mantém com outro sujeito (ou genericamente com outra parte). Tais modelos formam-se a partir de valores, arquétipos cognitivos e experiências que orientam para a adoção de determinadas atitudes e comportamentos.

De referir, ainda, que nas diversas interações, sejam na esfera institucional ou não, e independente do respeito e observância de normas sociais, normativos legais ou outros que existam formalizados, é facilmente percetível que a relação quotidiana é orientada pelo contrato psicossociológico onde as promessas não são necessariamente explícitas nem os deveres necessariamente escri- 
tos. Antes, atende a um conjunto de expectativas recíprocas relativas às obrigações mútuas entre cada indivíduo e a outra parte com quem estabelece o contrato psicológico (Paraíso, 2012). Como defendem Herriot e Pemberton (1997) "Psychological contrating is the social process whereby [the] perceptions are arrived at. (p.45).

Muito embora na literatura não se encontre uma concetualização universal, a natureza percetual e idiossincrática do contrato psicológico tem sido evidenciada ao longo de diversos estudos, mesmo os que se realizam para além do contexto organizacional. Razão que nos leva a uma abordagem do constructo com a acuidade devida pelo desconhecimento prévio da ambiguidade quanto à natureza das relações que estejam implícitas, assim como pela incerteza quanto ao futuro de tais interações ou mesmo dos contextos em que aquelas ocorrem.

Atendendo à natureza percetiva do contrato psicológico poder-se-á questionar, desde logo, quanto ao estado emocional com que aquela é apreendida. Goleman (2010) defende que é a emoção que guia o indivíduo. Nas suas palavras, "cada emoção representa uma diferente predisposição para a acção; cada uma delas aponta-nos numa direcção que já noutras ocasiões resultou bem para enfrentar o mesmo tipo de problema" (p.26).

Importa referir a relevância da capacidade de cada Ser de expressar as suas emoções e sentimentos, enquanto experiências mentais do foro subjetivo (Schultz \& Schultz, 2005), para melhor ajustamento entre si e as suas circunstâncias. A literatura tem evidenciado bem tal questão ao colocar a inteligência emocional como fator intrincado na abordagem ao comportamento humano. Como Mayer e Salovey (1997), citados por Gomes et al. (2008), a inteligência emocional representa a "capacidade de percepcionar as emoções, para aceder e gerar emoções que possam auxiliar o pensamento, para compreender as emoções e o conhecimento emocional, e para reflexivamente regular as emoções de tal modo que possam promover o crescimento intelectual e emocional" (p. 246).

A emergência de uma necessidade rompe um estado de equilíbrio do organismo provocando estados de tensão, insatisfação, desconforto e desequilíbrio (Paraíso, 2012).

Perante situações de dissonância, o indivíduo exerce uma ação ou comportamento capaz de aliviar a tensão gerada, ou mesmo, eliminar a situação de desconforto e desequilíbrio (Novaes, 2007; Paraíso in Veiga, 2016/2017).

Nesta eventualidade uma questão se coloca: como intervir? Fisher e Adams (1994), na sua perspetiva pragmática da comunicação humana, afirmam que os comportamentos, enquanto ações humanas observáveis, são fundamentais para compreender a comunicação. Subjacente está o princípio de que em qualquer interação - sequências de comportamentos num sistema interpessoal -, não se pode não comunicar. Mais! A comunicação é um fluxo contínuo de comportamentos interrelacionados que ligam indivíduos entre si. Surge no âmbito de determinados contextos, sob influências múltiplas, podendo circunstancialmente ocorrerem situações de rejeição, retroação ou, inclusivamente, a não integração do conteúdo da mensagem (Magno, 2014).

A linguagem humana, nas suas diversas vertentes, é uma característica própria do indivíduo. Uma corrente literária mais mentalista considera que o pensamento se assemelha a uma linguagem, uma vez constituído por símbolos ou representações mentais com uma sintaxe e semântica combinada numa característica peculiar: representação e interpretação dos factos percecionados como realidade.

Assim, a comunicação ganha contornos de imprescindibilidade nas relações interpessoais na medida em que é alavanca para o comprometimento individual (seja de natureza normativa, afetiva ou instrumental) determinando o grau do seu vínculo, bem como a sua manutenção ao longo do tempo (Paraíso, 2012). 


\section{CONTRATO PSICOLÓGICO: FORMALIZAÇÃO DA LINGUAGEM}

\section{OBJETIVOS DA INVESTIGAÇÃO}

A literatura compulsada, de onde se destacam autores como Rousseau $(1989,1995)$ e Guest (2004), apresenta modelos do contrato psicológico em contextos específicos, como é o caso do ambiente organizacional, assim como instrumentos metodológicos de acesso aos dados aplicáveis na relação de trabalho. Não descurando a conformidade de cada um, não encontramos nenhum estudo que apresente um modelo que registe, leia, interprete e devolva um sistema de informação que considere todas as formas de comunicação e não esteja dependente do tempo ou espaço para recolha de dados, ou seja, um sistema que registo qualquer forma de expressão do indivíduo a todo o tempo.

No presente trabalho temos como objetivo basilar realizar um estudo que sustente 0 desenvolvimento de um novo modelo aplicado ao contrato psicológico em qualquer circunstância em que este se desenvolva, que atente à realidade percecionada nas diversas interações e que, num sistema de informação, dê corpo às respostas dos sujeitos que aí atuam dinamicamente. Para tal é nosso entendimento ser fulcral estabelecer uma linguagem formal do modelo proposto.

\section{MÉTODO}

Já à época do apogeu da filosofia grega era entendimento que ninguém percebe uma suposta realidade. Antes, temos uma perceção desta filtrada pelas emoções, cultura, experiências e aprendizagens. Não obstante é possível estabelecer territórios limítrofes ambivalentes à subjetividade individual. Este é o grande desafio do investigador: desenvolver um interface entre 0 que aparenta ser unanimemente verdade daquilo que é subjetivamente interpretado como real e estabelecer uma correspondência apropriada entre ambas.

Nesta fase do Projeto adotamos uma postura analítica essencialmente de cariz concetual, a partir de uma abordagem qualitativa, que estuda os requisitos da linguagem formal pelo desenvolvimento de uma gramática própria associada a um modelo de genérico de contrato psicológico pela sistematização da informação.

\section{RESULTADOS ALCANÇADOS}

Na revisão da literatura efetuada concluímos que o contrato psicológico é um acordo implícito, não formal, baseado em promessas, resultantes de crenças individuais, cruciais na moldagem das atitudes e dos comportamentos dos sujeitos nas suas interações com as partes às quais estão (em determinado período) vinculados. Estudos teóricos e empíricos têm evidenciado que a perceção de incumprimento do contrato psicológico, por parte de um indivíduo, tem implicação ao nível das atitudes e comportamentos.

0 modelo teórico do contrato psicológico criado e representado pela Figura 1, que serve de base à presente investigação, observou os fundamentos na teoria de Rousseau (1989), Guest (2004), Paraíso (2012) e Magno (2014).

No essencial o nosso modelo do contrato psicológico observa:

um conjunto de fatores contextuais individuais e externos;

um conjunto de variáveis intervenientes e moderadoras decisivas na apreensão da suposta realidade, com repercussão na avaliação que o sujeito faz dos termos do seu contrato psicológico;

as diferentes implicações nas atitudes e nos comportamentos de cada indivíduo em função da comparação entre o que 0 indivíduo espera e apreende como resultado na interação com a outra parte no contrato;

o sistema de informação que agrega, numa linguagem informal, a atribuição de sentido (resposta); 
a sistematização de informação após a interpretação e (re)significação semântica da simbologia da linguagem do contrato psicológico.

Figura 1:

Modelo teórico do contrato psicológico

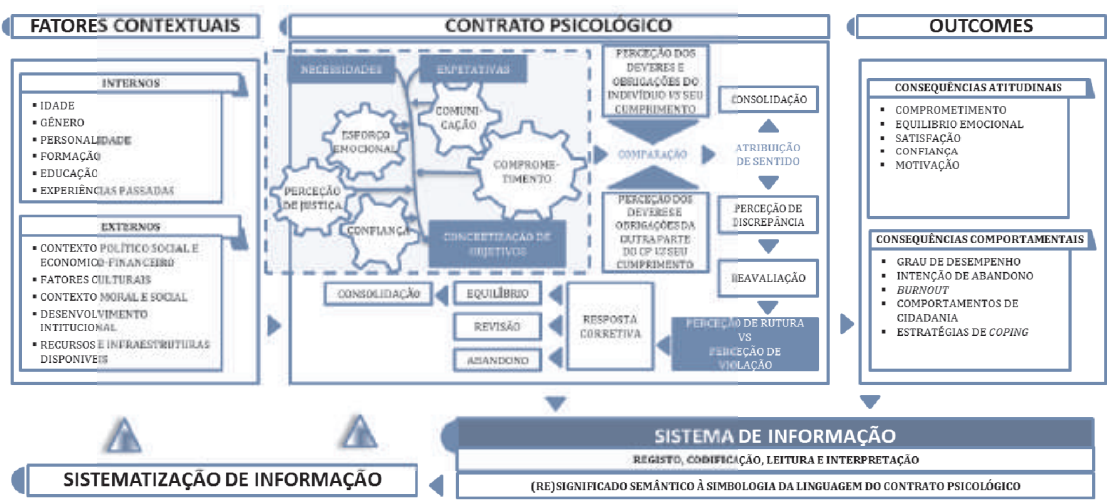

De acordo com Scotti (1994), "Noam Chomsky é um dos teóricos que mais claramente propôs as possíveis e prováveis relações entre o fenómeno da linguagem e correlatos processos mentais" (p.64). Já Vygotski considera que a linguagem tem uma estrutura funcional e instrumental na medida em que possibilita a interação entre si. Perspetiva que compreende a linguagem como elemento presente nas relações do pensamento.

Ora, como advoga Bernardes (2008), para que o pensamento seja inteligível e comunicável "necessita de ser transformado em um sistema de códigos instituído socialmente que expresse as suas relações semânticas” (p.71).

Com o propósito de definir as regras de comportamento do modelo do contrato psicológico evidencia-se apenas a componente fulcral do modelo anterior - modelo proposicional do contrato psicológico -, conforme Figura 2, onde as proposições estabelecem as regras de transição entre as variáveis da investigação.

Figura 2:

Modelo proposicional do contrato psicológico

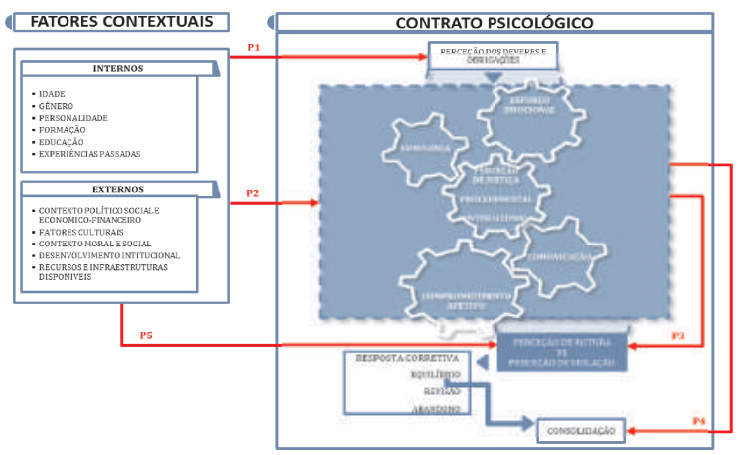




\section{CONTRATO PSICOLÓGICO: FORMALIZAÇÃO DA LINGUAGEM}

Assim, podemos definir, informalmente, uma linguagem como sendo um sistema desejável para expressar determinadas ideias, factos ou conceitos, incluindo um conjunto de símbolos e regras para a sua manipulação. Já uma linguagem formal é um mecanismo formal de representação e especificação de uma linguagem. Tal pode ser reconhecida por um autómato ou gerada por uma gramática, de onde se destacam as gramáticas de Chomsky.

Formalmente, a gramática geradora de uma linguagem é definida pelo quádruplo ordenado $G=$ ( $V, T, S, P R O D)$, onde:

$V$ e $T$ são conjuntos finitos não vazios disjuntos onde $V$ é o conjunto dos símbolos não terminais ou, apenas, designadas variáveis e Té o conjunto dos símbolos terminais. Da reunião dos conjuntos $V$ ou $T$ resulta o vocabulário da gramática;

$S$ é uma variável designada por axioma da gramática ou, simplesmente, símbolo inicial;

$P R O D$ é um conjunto finito não vazio de regras de reescrita ou, simplesmente, designadas por produções.

Passamos, agora, a especificar uma gramática GCP geradora da linguagem do contrato psicológico $L(G C P)$. Assim, de acordo com o modelo proposicional observa-se que os fatores contextuais (internos e externos) que constituem o conjunto $F C$, as proposições $\left(\mathrm{P}_{1}, \ldots, \mathrm{P}_{\mathrm{j}}\right)$ que definem as regras do comportamento do modelo que compõem o conjunto $P P$, as variáveis intervenientes (perceção de deveres e obrigações, atribuição de sentido) que formam o conjunto VI, as variáveis moderadoras (esforço emocional, perceção de justiça, confiança, comunicação e comprometimento) que compõem o conjunto VMe as variáveis transitórias (quebra -perceção de rutura vs perceção de violação- e equilíbrio) que formam o conjunto $V T$ agregam as variáveis da gramática GCP.

Por outro lado, as descrições das proposições de $P P$ que compõem o conjunto $D P$, os enunciados das proposições de $P P$ que formam o conjunto $E P$, as perceções expressas pelo indivíduo relativamente a cada proposição de $P P$ que constituem o conjunto $P R$, bem como as classificações destas perceções que compõem o conjunto $C L$ constituem os símbolos terminais da gramática GCP. São ainda símbolos terminais da gramática a categorização de cada uma das variáveis de VI e de VM que compõem o conjunto $C T$, as variáveis de resposta corretiva (consolidação, revisão e abandono) que formam o conjunto $R C$ e, finalmente, as perceções gerais do indivíduo em relação às respostas corretivas de $R C$ que constituem o conjunto $P G$.

Assim, podemos definir uma gramática geradora da linguagem do contrato psicológico $L(G C P)$ formalmente por:

$$
\begin{aligned}
G C P= & (V=\{S\} \cup F C \cup P P \cup V I \cup V M \cup V T \\
& T=D P \cup E P \cup P R \cup C L \cup C T \cup R C \cup P G, \\
& S \\
& P R O D)
\end{aligned}
$$

onde:

PROD é o conjunto de produções definidas por: 
i. $\quad S \rightarrow$ FATOR_CONTEXTUAL

$\forall$ FATOR_CONTEXTUAL $\in$ FC

ii. S $\rightarrow$ FATOR_CONTEXTUAL PROPOSICAO VARIAVEL

$\forall($ PROPOSICAO : FATOR_CONTEXTUAL $\rightarrow$ VARIAVEL $) \in P$ P, COm FATOR_CONTEXTUAL $\in$ FC e VARIAVEL $\in$ VI U VMUVT

iii. FATOR_CONTEXTUAL $\rightarrow$ categoria FATOR_CONTEXTUAL

$\operatorname{com} \boldsymbol{F}$.

iv. FATOR_CONTEXTUAL $\rightarrow$ categoria

com $F$

v. FATOR_CONTEXTUAL $\rightarrow$ percecao_geral consolidacao

com percecao_geral $\in P G$

vi. FATOR_CONTEXTUAL $\rightarrow$ percecao_geral revisao

com percecao_geral $\in P G$

vii. FATOR_CONTEXTUAL $\rightarrow$ percecao_geral abandono

com percecao_geral $\in P G$

viii. PROPOSICAO $\rightarrow$ descricao enunciado

com descricao $\in$ DP e enunciado $\in$ EP

ix. VARIAVEL_INDEPENDENTE

$\rightarrow$ representacao classificacao VARIAVEL_MODERADORA

$\operatorname{com} V_{t}$ classificacao $\in$ CL e VARIAVEL_MODERADORA $\in$ VM

x. VARIAVEL_MODERADORA $\rightarrow$ representacao classificacao consolidacao COM VARIAVEL_MODERADORA $\in$ VM, representacao $\in P R$ e classificacao $\in \boldsymbol{C L}$

xi. VARIAVEL_MODERADORA $\rightarrow$ representacao classificacao QUEBRA COM VARIAVEL_MODERADORA $\in$ VM, representacao $\in P R$ e representacao $\in C L$

\footnotetext{
xii. QUEBRA $\rightarrow$ EQUILIBRIO

xiii. QUEBRA $\rightarrow$ revisao

xiv. QUEBRA $\rightarrow$ abandono

xv. EQUILIBRIO $\rightarrow$ consolidacao
} 


\section{CONTRATO PSICOLÓGICO: FORMALIZAÇÃO DA LINGUAGEM}

A título de exemplo de uma frase da linguagem $L(G C P)$ considere-se uma proposição $P 2 a$ enunciada nos seguintes termos: "Os indivíduos evidenciam maior confiança na relação que estabelecem com o seu superior hierárquico quando maior a idade deste". Considere-se agora a expressão de um indivíduo de 35 anos: "(...) a experiência que o meu chefe revela dá-me confiança e segurança no desempenho das minhas funções diárias (...). Confio nele cegamente".

A sequência geracional, usando GCP, da perceção desse indivíduo pode ser descrita por:

$S \rightarrow$ IDADE P2a CONFIANÇA

(ii)

$\rightarrow$ “[30-40] anos” P2a CONFIANÇA

(iv)

$\underset{\text { (viii) }}{\rightarrow}$ "[30-40] anos" "P2a" "Os indivíduos evidenciam maior confiança na relação que

estabelecem com o seu superior hierárquico quando maior a idade deste” CONFIANÇA

$\vec{~} \vec{x}$ “[30-40] anos" "P2a" "Os indivíduos evidenciam maior confiança na relação que (x)

estabelecem com o seu superior hierárquico quando maior a idade deste" "Confiança no chefe ..."

"Confiança no superior" consolidacao

\section{CONCLUSÕES}

Não há intervenção consciente se não houver conhecimento sobre 0 universo que nos rodeia. Do mesmo modo, não se poderá compreender o comportamento humano se os contextos em que aquele ocorre forem descurados, sendo certo que o comportamento não está isento da influência de motivos disposicionais ou da apreensão e interpretação que cada um faz acerca da realidade que perceciona.

Da complexidade circunstancial, que cruza variáveis multidimensionais intrínsecas e extrínsecas a cada sujeito, resultam estados que motivam atitudes e comportamentos passíveis de expressão pelos próprios numa linguagem natural ou informal.

Num quotidiano vivido a ritmos e sentidos desconcertantes urge a compreensão da relação entre o pensamento e a linguagem que, na teoria de Vygotski, são a chave para compreender a natureza da consciência humana, numa realidade socialmente construída.

0 conhecimento das respostas individuais (dados empíricos), percecionados pela consciência subjetiva ou manifestados em estado de escape emocional, vertidos num sistema de classificação e categorização, possibilita a constituição de um conjunto de informação que reflete os significados atribuídos pelos sujeitos.

Através da gramática do contrato psicológico especificámos as bases de uma nova linguagem para o conhecimento conquistado, pela voz dos sujeitos, a linguagem do contrato psicológico aplicado a qualquer contexto.

A gramática estipulada tem uma correspondência direta com o modelo teórico do contrato psicológico definido numa articulação dinâmica e evolutiva. Com efeito, tal evolução da linguagem resulta de uma construção adaptativa à introdução de novos componentes no próprio modelo, ou de novas codificações, enquanto processo de reajustamento às mudanças contextuais ou às experiências vivenciadas pelos indivíduos.

Refira-se, igualmente, que o presente trabalho tem, ainda, como contributo a preocupação e 0 desejo de descobrir "segredos" da linguagem humana através da realização de uma análise semio- 
lógica pela continuidade e descontinuidade semântica, aspeto pouco instigado nos estudos contemporâneos.

De resto, como escreveu o Poeta em "O Mistério das Cousas"

0 mistério das cousas, onde está ele?

Onde está ele que não aparece

Pelo menos a mostrar-nos que é mistério?

(...) Sempre que olho para as cousas e penso no que os homens pensam delas,

(...) Porque o único sentido oculto das cousas

É elas não terem sentido oculto nenhum,

É mais estranho do que todas as estranhezas

$E$ do que os sonhos de todos os poetas

E os pensamentos de todos os filósofos,

Que as cousas sejam realmente 0 que parecem ser

E não haja nada que compreender.

Sim, eis o que os meus sentidos aprenderam sozinhos: -

As cousas não têm significação: têm existência.

As cousas são o único sentido oculto das cousas.

Fernando Pessoa

\section{REFERÊNCIAS BIBLIOGRÁFICAS}

Bernardes, M. E. M. (2008). Transformação do pensamento e da linguagem na aprendizagem de conceitos. Psicologia da Educação, 26, 67-85.

Correia, R., \& Mainardes, E. W. (2010). 0 desenvolvimento do contrato psicológico orientado para desempenhos de elevado rendimento. PSICO, 41, 266-277.

Côrtes, L. L., \& Silva, J. R. G. (2006). Construção de contratos psicológicos de indivíduos que ingressam em organizações do sector público no atual contexto brasileiro: Estudo de caso de uma empresa estatal. Paper presented at the $30^{\circ}$ Encontro da ANPAD, Salvador, Brasil.

Fisher, B. A., \& Adams, K. L.(1994). Interpersonal Communication: Pragmatics of Human Relationships. McGraw-Hill.

Goleman, D. (2010). Inteligência emocional (15 ed.). Lisboa: Círculo de Leitores.

Guest, D. E. (2004). Flexible employment contracts, the psychological contract and employee outcomes: An analysis and review of the evidence. International Journal of Management Reviews, 5, 1-19.

Herriot, P. \& Pemberton, C. (1997). Facilitating new deals. Human Resource Management Journal, 7, 45-56.

Gomes, J. F., Cunha, M., Rego, A., Cunha, R., Cabral-Cardoso, C., \& Marques, C. A. (2008). Manual de gestão de pessoas e do capital humano. Lisboa: Edições Sílabo.

Magno, J. (2014). Contrato Psicológico na Relação Professor-Aluno no Ensino Superior. (Tese de doutoramento), Departamento Psicología y Antropologia : Universidade de Extremadura, Badajoz.

Novaes, M. V. (2007). A importância da motivação para o sucesso das equipes no contexto organizacional. Revista Científica de Psicologia, 1(1), 130-150.

Paraíso, I. (2012). Política de austeridad y contrato psicológico en la Administración Pública. (Tese de doutoramento), Departamento Psicología y Antropologia : Universidade de Extremadura, 


\section{CONTRATO PSICOLÓGICO: FORMALIZAÇÃO DA LINGUAGEM}

\section{Badajoz.}

Robinson, S., \& Rousseau, D. (1994). Violating the psychological contract: not the exception but the norm. Journal of Organizational Behavior, 15, 245-259.

Rousseau, D. M. (1989). Psychological and implied contracts in organizations. Employee Responsibilities and Rights Journal, 2(2), 121-139.

Rousseau, D. M. (1995). Psychological contracts in organizations: Understanding written and unwritten agreements. Thousand Oacks: SAGE.

Schultz, D., \& Schultz, S. (2005). História da psicologia moderna (8 ed.). São Paulo: Thomson.

Scotti, S. (1994). A linguagem e os processos mentais. Revista de Ciências Humanas, 12.6, 63-76.

Veiga, F. H. (Orgs), Pereira, A., Carvalho, C., Goulão, F., Marinha, F., Oliveira, I., Faria, L., Taveira, C., Bahia, S., Raposo, S., \& Caldeira, S. (2016/2017). Atas do II Congresso Internacional Envolvimento dos Alunos na Escola - Perspetivas da Psicologia e Educação, Motivação para 0 Desempenho Académico. Lisboa: Instituto de Educação da Universidade de Lisboa. 